\title{
A Scientific Review on Various Pellet Extruders Used in 3D Printing FDM Processes
}

\author{
Yousuf Pasha Shaik, Jens Schuster, Aarif Shaik \\ Institute for Polymer Technology West-Palatinate, University of Applied Sciences Kaiserslautern, Pirmasens, Germany \\ Email: yousufpashashaik@gmail.com
}

How to cite this paper: Shaik, Y.P., Schuster, J. and Shaik, A. (2021) A Scientific Review on Various Pellet Extruders Used in 3D Printing FDM Processes. Open Access Library Journal, 8: e7698.

https://doi.org/10.4236/oalib.1107698

Received: June 25, 2021

Accepted: July 26, 2021

Published: July 29, 2021

Copyright $\odot 2021$ by author(s) and Open Access Library Inc.

This work is licensed under the Creative Commons Attribution International License (CC BY 4.0).

http://creativecommons.org/licenses/by/4.0/

(c) (i) Open Access

\begin{abstract}
3D Printing is the trending technology in this generation and its usage is increasing day-by-day because it reduces cost of production. FDM (Fused Deposition Modeling) is one type of 3D printing which uses plastics in the form of wire like filament by an extruder for making 3D prints. But, by installing a pellet extruder instead of filament extruder reduces cost of printing process that is $1 \mathrm{~kg}$ of PLA pellets cost around $4 €$ while $1 \mathrm{~kg}$ of PLA filament cost around 22€; also, can print wide ranges of materials, increase in speed of prints, chance of recyclability when compared to filament extruder. Whole purpose of this paper is to provide information about installation of a miniature pellet extruder to a 3D printer especially in FDM 3D PRINTERS instead of filament extruder. Most of the FDM 3D printers use plastics in the form of wire like filament for making 3D prints. This paper provides in detail information about FDM and its types, difference between pellet extruder and filament extruder, their advantages and disadvantages, information about some pellet extruders and type of FDM printers they are suitable for. Knowledge from this study will help to choose between pellet extruder and filament extruder which fulfils requirement and which types of pellet extruder have to install to $3 \mathrm{D}$ printer depending on its type and requirement.
\end{abstract}

\section{Subject Areas}

Mechanical Engineering

\section{Keywords}

Additive Manufacturing, FDM, Mechanical Properties Pellet Extruder, PLA

\section{Introduction}

3D printing is also termed as Additive Manufacturing. In earlier 1980s, additive 
manufacturing was mostly used for the manufacturing of prototypes, but, in these years, their usage has increased due to their precision, repeatability and production costs. Depending upon their usage, there are many types of 3D printing. Some major types are SLA (Stereolithography), FDM/FFF (Fused Deposition Modeling/Fused Filament Fabrication), Material Extrusion, Binder Jetting, Powder Jetting, Direct Energy Deposition, etc. Among all types of 3D Printings, FDM is the most effective technique to print prototypes as well as custom thermoplastic components [1].

Fused Filament Fabrication (FFF) is a building process in which material is melted and depositing layer by layer to manufacture a compound as shown in Figure 1 and the entire layering process from top to bottom is shown in Figures 1 (a)-(c). This phenomenon is carried out in a specific manner by a slicing program. The material which uses in FFF is in form of filament which is made from ant type of plastic. The name FDM and FFF, when we consider about the procedure, there is not much difference but when we look at deep level, some differences are there which explained below and there is a historical background about these names [2].

FDM is 3D printing technology which was developed in 1989 by Stratasys. According to them, a 3D printer consists of some important parts like a feeder, an extruder, a nozzle, and the process is feeder that feeds the filament of plastic and extruder nozzle extrudes it by melting the filament in the heat sink. The Stratasys Company has the FDM patent rights until 2009. So, the RepRap community which found in 2005, had come with an alternative FFF (Fused Filament Fabrication) which also gives the same meaning with some minute change with FDM (Fused Deposition Modeling) [2].

\section{Types of FDM 3-D Printers}

\subsection{Cartesian 3D Printer}

In 3D Printing market, most of the FDM 3D Printers works on a principle of Cartesian coordinate system. In Mathematics, Cartesian coordinate system means to locate a point with the help of distances in $\mathrm{X}, \mathrm{Y}$ and $\mathrm{Z}$ axis. This movement in all axis direction involves movement of printhead and printing bed. In most of the FDM 3D Printers, the bed plate moves in Z-axis and printer

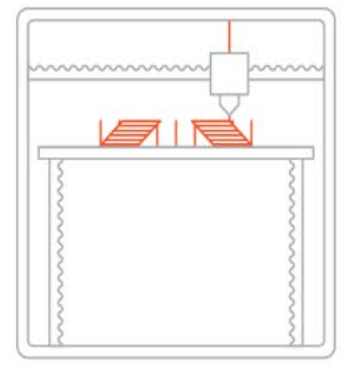

(a)

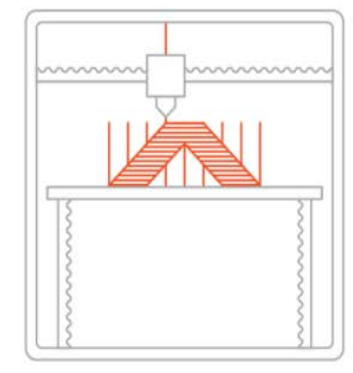

(b)

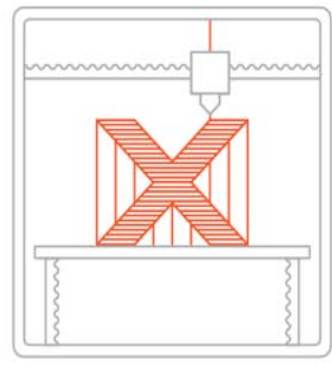

(c)

Figure 1. FDM 3D printing process. 
head moves in $\mathrm{X}$ and $\mathrm{Y}$ direction. In some printers, they have only $\mathrm{Y}$-axis direction for print build plate and printhead moves in $\mathrm{X}$ and $\mathrm{Z}$ directions [3] as shown in Figure 2.

\subsubsection{SCARA 3D Printer}

SCARA means Selective Compliance Articulated Robot Arm. These FDM 3D Printers also works on the principle of Cartesian coordinate system. But their difference from other Cartesian 3D Printers are their arrangement of their parts and their working mechanisms.

As shown in Figure 3, SCARA 3D Printers have a robot arm with printhead fixed to it. It consists of two motors which are used for the movement of the robotic arm, each for the movement of printhead in XY plane and movement $\mathrm{Z}$ Plane [4].

\subsubsection{Delta 3D Printer}

The most significant FDM 3D Printer that seen in market is Delta 3D Printer. Its latest edition, which was developed by swiss students that can work in six-axis. These Delta machines also follows Cartesian coordinate system. It includes a round printing plate which is a connected to an extruder. This extruder is fixed to their triangular point, its position and direction are depending on the up and down movement of the three joints as shown in Figure 4.

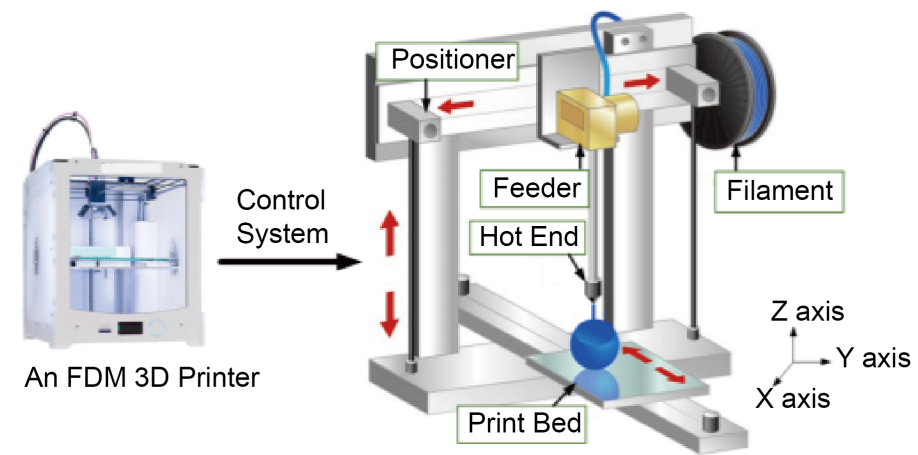

Figure 2. Cartesian printer.

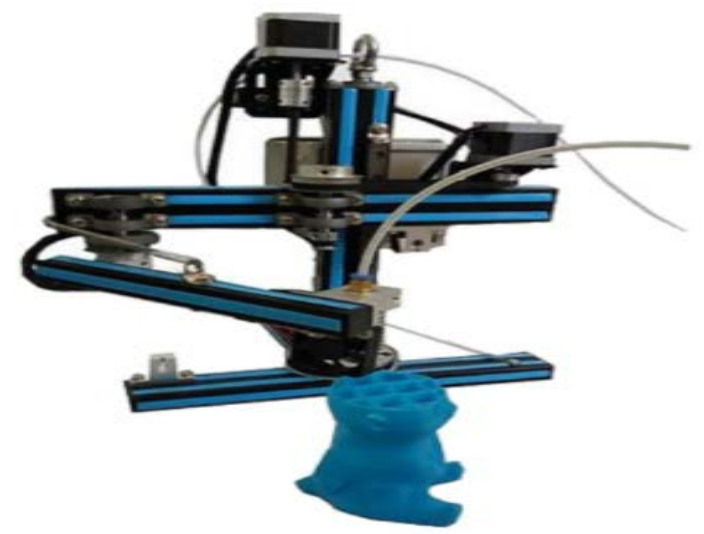

Figure 3. SCARA type. 


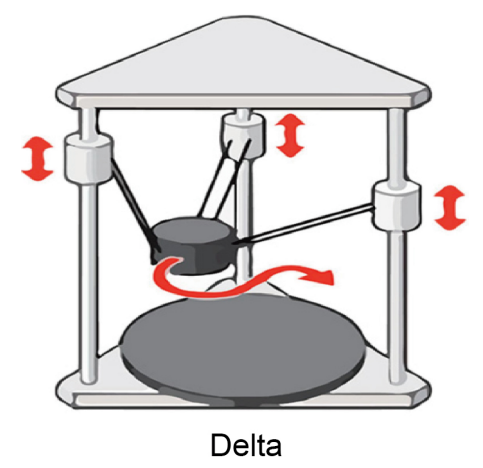

Figure 4. Delta type.

The main purpose of the Delta 3D Printers is to fast up the printing process as quick as possible. But it is believed that accuracy of Delta 3D Printers is far less than conventional Cartesian Printers. Even though Delta's printers follow Cartesian coordinate system but they are different from Cartesian printers. The difference is only in where each element can move in relation to the print bed [3].

\subsubsection{H-Bot 3D Printer}

H-bot 3D Printers are different because of their arrangement of belts used to move printhead within the XY Plane. In this 3D Printer, printhead moves in $\mathrm{X}$-axis direction with the help of one belt and with the help of another belt, the printhead moves in $\mathrm{Y}$-axis. The print bed moves up and down direction in $\mathrm{Z}$-axis direction iluustrated in Figure 5.

These H-bot printers are more similar with Core-XY 3D Printers, which results confusion between them. To differentiate them, Core-XY 3D printers make use of an alternative belt system. In most cases, they can easily be distinguished by the shape of their belts when looked at from above. An H-bot's belts will form, an ' $\mathrm{H}$ ', while a Core-XY forms more of a boxy " $\mathrm{A}$ ” [4].

\subsubsection{Core-XY 3D Printer}

In Core XY 3D Printers, the movement of printhead is carried with the help of arrangements of pulleys and belts in XY plane, as similar as in H-bot Printers. Core XY printers move one belt each to position the print head by using two stepper motors depicted in Figure 6.

The print bed is mounted on a Z-axis motion system, such as threaded rods. The print bed moves up and down by using this arrangement, so that the printhead can print layers for the desired part. To contain axes and pulleys the Core $\mathrm{XY}$ printers are usually cube-shaped [4].

\subsubsection{Conveyor Belt 3D Printer}

With respect to the printhead, most belt printers are essentially Core XY printers with one of the top edges tilted downwards. Then, replacing the print surface is a sort of conveyor belt, which moves one way or the other in the combined Z- and 


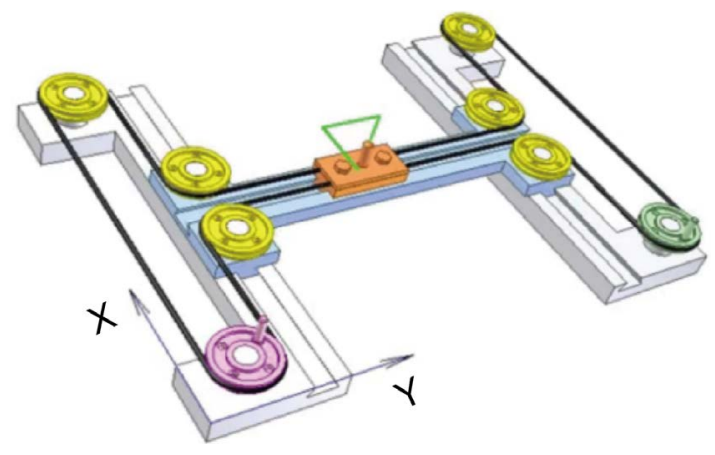

Figure 5. H-Bot type.

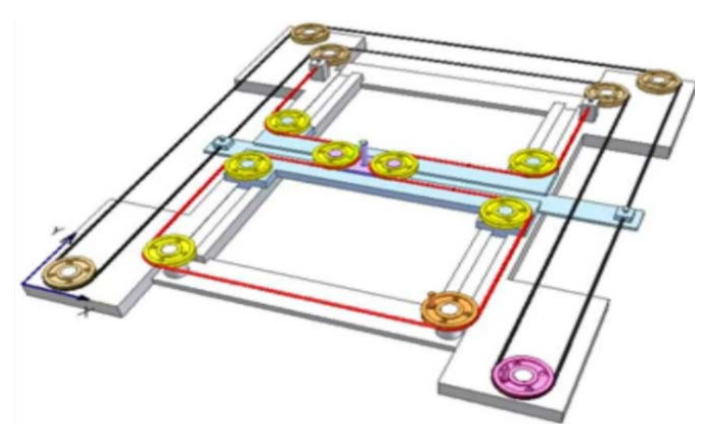

Figure 6. Core-XY type.

Y-axes. This belt can also print an infinitely long piece or continuously many small parts.

Typically belt $3 \mathrm{D}$ printers have the $\mathrm{Z}$-axis at a 45 -degree angle, which allows for printing taller. With this, the printhead moves up and down as well as left and right. However, some belt 3D printers incorporate alternative motion styles too [4].

\subsubsection{Polar 3D Printer}

Polar 3D printers are different from the other FDM which makes use of the polar coordinate system. Polar coordinate system, meaning it plots points on a circular grid rather than a rectangular one. Polar printers use angle and distance to locate points in two dimensions (on the XY plane). Polar printers use a spinning circular build plate. As the mounted print head arm moves up along the $\mathrm{Z}$-axis the machine prints the desired object layer by layer by using this motion [4].

\subsubsection{Robot Arm 3D Printer}

In huge automobile plants robot arms are used for assembly purpose. On an extension its usage robot arms are began to be using in $3 \mathrm{~d}$ printing also for production purpose, also in construction of homes and buildings, this type of $3 \mathrm{~d}$ printing with robot arm is still in developing stage.

$3 \mathrm{D}$ printing with robot arm is not commonly used compared to other 3D printing techniques because of its cost and it requires space. But usage of robot arm is increasing because its flexibility and not a fixed one which making it more user friendly. Due to its flexibility, one can move the printer head in any direc- 
tion, which enables to print complex structure easily without any difficulty, but the printing quality is not as good as other cartesian printers when compared [3].

\section{Types of Extruders based on Feeding Forms}

Main component in all FDM 3D printers is Extruder, which plays a crucial role in effective working. An extruder is a device which heats and melts the feed and extrudes through the nozzle by following the defined path on the print bed. Some important types of extruders based on their feeding forms as follows:

- Filament extruder.

- Pellet/Granules extruder.

\subsection{Filament Extruder}

Before going to discuss about working of filament extruders, we must know some important parts in extruders and their importance. They are as follows:

\section{Stepper Motor:}

Stepper motor works by means of electricity and most commonly in a range of $12 \mathrm{v}-24 \mathrm{v}$. Its function is to rotate small gear which in turn rotates the large gear as shown in figure. This phenomenon leads to the feeding of filament to the extruding end [5].

\section{Cooling Fan:}

Cooling fans help to improve the quality of the printed object to be consistent and as well as less depends on temperature around extruder to get a quality product by helping solidify the object in fraction of time. Bridging performances are also improved as well [5].

\section{Hot end and Cold end:}

In most of the FDM 3D Printers have hot end and cold end. Cold end is the upper part of extruder before entering into the heat sink where it is pushes into the heat sink with the help of electric motor and hot end is present between heat sink and nozzle where it heats and melts the filament [5].

\section{Filament:}

In most of the 3D Printers, filaments like PLA, ABS, Polypropylene etc., are used commonly. Mostly they are of in $85 \mathrm{~mm}$ diameter. Smaller the diameter then higher the printing quality is achieved [5].

\section{Heat sink:}

Heat sink is designed in such a way that has fins on its outer part and helps to release heat from the heat block as shown in Figure 7 and helps to prevent pre-heating of filament which may leads to the overflow. Aluminium is commonly used in for their manufacturing [5].

\section{Heat Breaker:}

Heat breaker is mostly made of titanium as well as stainless steel because it a poor conductor of heat and it main purpose is to transition state as short as possible which helps the filament to be in a molten state as little as possible to 


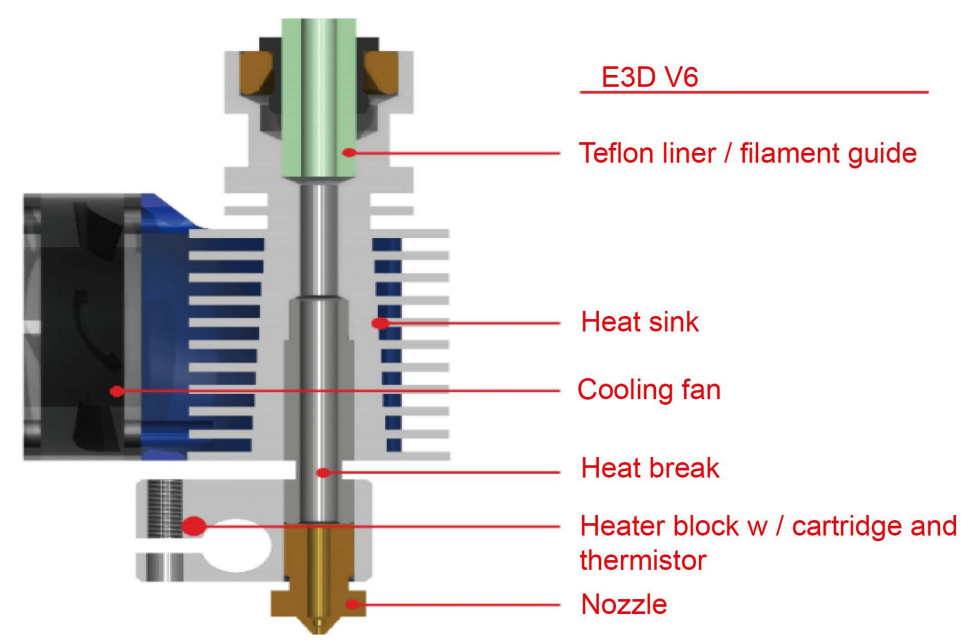

Figure 7. Filament extruder cross-section.

prevent overflow. Heat breaker is a connection between heat sink and heat block [5].

\section{Heat Block:}

Usually made from aluminium, the heater block joins the nozzle to the heat break and holds the heater cartridge and thermistor [5].

\section{Thermistor:}

This part senses the temperature of the heat block. It is usually a small glass bead with two wires attached (which are typically insulated with glass fiber or Teflon). For high-temperature printing, a thermocouple may be used instead [5].

\section{Build plate:}

Build plate is an open-frame design and platform where the printing take place below the extruder nozzle. The plate must be coated with glass, metal, or ceramic material. In some printers, the build plate may be heated or may not heated [5].

\section{Teflon Liner:}

Some hot ends have a PTFE liner that guides the filament through the heat break into the nozzle. This makes it easier to print PLA, but the temperatures at which you can print is limited as compared to all-metal hot end (making it difficult to print PETG and impossible to print polycarbonate) [5].

\section{Working process:}

In a filament extruder, an extrusion head is used for the fabrication of material by unwinding plastic filament which is known as hot head. Feeding of filament to the hot head is in a controlled rate by the hot drive.

When the filament enters the nozzle, it melts after it reaches the glass transition temperature due to heat by the heater in the hot end. The most important requirement is filament must be a thermoplastic to be melted to fabricate the desired object.

As the melted filament exits the nozzles, it solidifies immediately under room temperature. If entire process is done in an inert gas chamber, it helps to prevent 
oxidation and improves mechanical properties of printed object because the inert gas improves the adhesion between the layers.

Depending upon the types of printer, the movement of extruder changes. Nozzle can move in $\mathrm{X}$ and $\mathrm{Y}$ plane and bed can moves in $\mathrm{Z}$ direction in some types of $3 \mathrm{~d}$ printers and some bed moves in $\mathrm{Y}$ direction and nozzle in $\mathrm{X}$ and $\mathrm{Z}$ direction. The stepper motor guides the nozzle in XYZ plane [6].

\subsubsection{Advantages of Filament Extruder}

- Quality of print: Printing quality and surface finish are very good.

- Oozing: In filament extruder problem of oozing is very low.

- Easy handling: Change of filament and operating are very easy to handle.

- Ability to print complex design: Printing of complex design with a detailed surface finish is possible because of using smaller size nozzles [6].

\subsubsection{Some Common Problems in Filament Extruder}

3D Printing with Filament extruder experiences several problems.

Warping: Bending at the edges of print just above the print bed, this phenomenon is known as Warping. Warping is mainly caused due to the plastic cools quickly. This problem is commonly seen in PLA and ABS. Best solution to prevent warping is using a heated printed bed maintained at Glass Transition Temperature [7].

Nozzle Height: Distance between the nozzle and print bed is called Nozzle Height. If nozzle touches the print bed, leads to blockage of melted filament in extruder [7].

Clogging: The blocking of filament inside the extruder which does not allow the further movement of molten plastic from the nozzle. This phenomenon is called Clogging. To prevent this problem, printing process always at right temperature [8].

Stringing/Oozing: When nozzle moves to a new location, the molten plastic forms hairy attachments leaving behind is called Oozing. This problem is because of overheating [9].

\subsection{Pellets/Granular Extruder}

The working mechanism of pellet extruder is as same as filament extruder, which uses pellets instead of filament. Some additional parts are present like Hopper and Auger screw. The screw helps to transfer pellets from the inlet to the nozzle opening. Before extruded through the nozzle, the melted plastic undergoes high pressure due to the screw which having transport zone, melting zone, and mixing zone. Pellets converts from solid form to liquid paste form by means of heater which heats the screw and barrel. The motor, which is connected to screw rotates the screw which in turn exerts high pressure to extrude as shown in Figure 8.

Screw speed is responsible for the exposure time of material to the heat sources. Problems due to high temperature like preheating of pellets in inlet, 


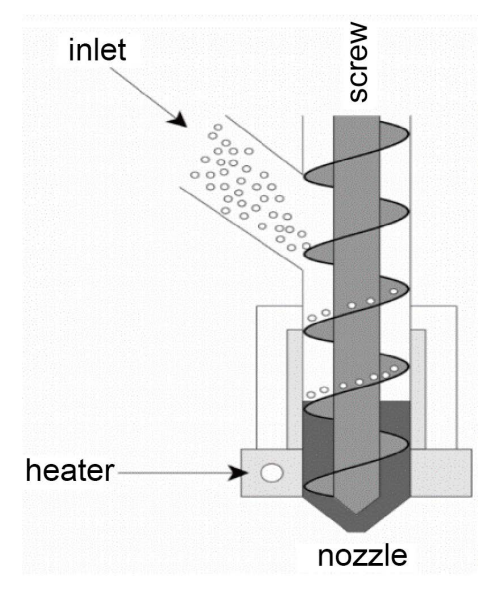

Figure 8. Pellet extruder.

which leads to entire system blockage and leads to burning of pellets results reduction in viscous nature at nozzle. As well as problems due to low temperature leads to jams which interrupts extrusion. An important point to be considered is smaller the pellet size higher the melting rate [10].

\subsubsection{Advantages of Pellet Extruder}

- Cost friendly: Manufacturing cost of product with pellet extruder is less when compared to filament extruder because the cost of pellets is very less than filament cost that is $1 \mathrm{~kg}$ of PLA pellets cost around $4 €$ while $1 \mathrm{~kg}$ of PLA filament cost around $22 €$ which results decrease in the production cost.

- Prints large parts: Most industries use pellet extruder to print large parts in less time with customizable materials. Because of having large nozzle sizes, flow rate is high results to print parts quickly.

- Wide range of materials: There is a chance of using large number of materials like most technical ones like polycarbonate (PC), TPU, EVA etc., which are more in cost in filament form and able to add loads of materials in pellet extruders.

- Recyclability: In pellet extruder, there is possibility to feed recycle plastic and from the part which was printed on same extruder.

- Reduction in printing time: Due to having large nozzle in pellet extruder, speed of materials output is high which results reduction in printing time when compared to filament extruder [11].

\subsubsection{Some Common Problems in Pellet Extruder}

In 3D printing with pellet extruder also experiences problems like filament extruder. They are Warping, Clogging, Porosity, Oozing, under extrusion-over extrusion, sink marks, Burn Marks, etc. Some of the major problems, their causes and solutions are discussed below:

Warping: The finished product had unwanted turns and bends is named as Warping. It is due to the low bed temperature than the plastic glass transition 
temperature. To prevent this phenomenon, bed temperature is maintaining at glass transition temperature and by applying adhesive to the print bed.

Porosity: Porosity is defined as when the final print contains voids and air bubbles, inside or outside. This problem is mainly caused due to the trapped air and moisture present in the pellets. To prevent this, pre-heating of pellets and use small pellets which allow to escape air etc.

Clogging: Blocking of extruder from inside which does not allow the further movement of molten plastic from the nozzle. This phenomenon is called Clogging. Clogging is mainly caused due to, if the hot end is at low temperature, the pellets melt improperly leads to the blockage of extruder. To prevent this problem, printing process should always be done at right temperature.

Under Extrusion-Over Extrusion: To prevent this problem pellets level should maintain properly and have to check continuously. Also have to maintain temperature depends on the type of plastic pellets.

\subsection{Powder Melt Extrusion}

It is modified version of pellet extruder which uses powder form of polymer rather than pellet. Usually in pellet extruders uses pellets in a range of $2 \mathrm{~mm}$ $4.5 \mathrm{~mm}$ but in powder melt extruder it is in a range of $0.038 \mathrm{~mm}-1 \mathrm{~mm}$. The main aim is to develop an extrusion process which does not require any pre-processing of powder granules to pellets which require in pellet extrusion. It gives the ability to the extruder to print directly from the powder granules, results no need of special equipment for cutting and extrusion equipment which reduces the printing cost. But the printing quality is comparatively less than pellet extruders, problem found that inconsistent extrusion and lower print resolution. This printing technique is still in developing stage [12].

\subsection{Dual Extruders}

Figure 9 illustrates, Dual extruders are having two nozzles, and which enables to print a part of two or more materials. The process is same as single extruder, but it switches automatically according to given instructions. Dual extruders help to print a part of different colors, different materials and support structures which saves a lot of time. Main disadvantage of dual extruder is their cost [13].

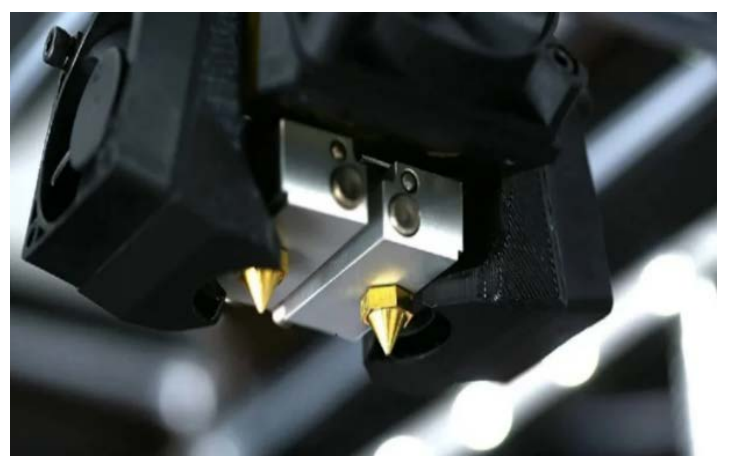

Figure 9. Dual extruder. 


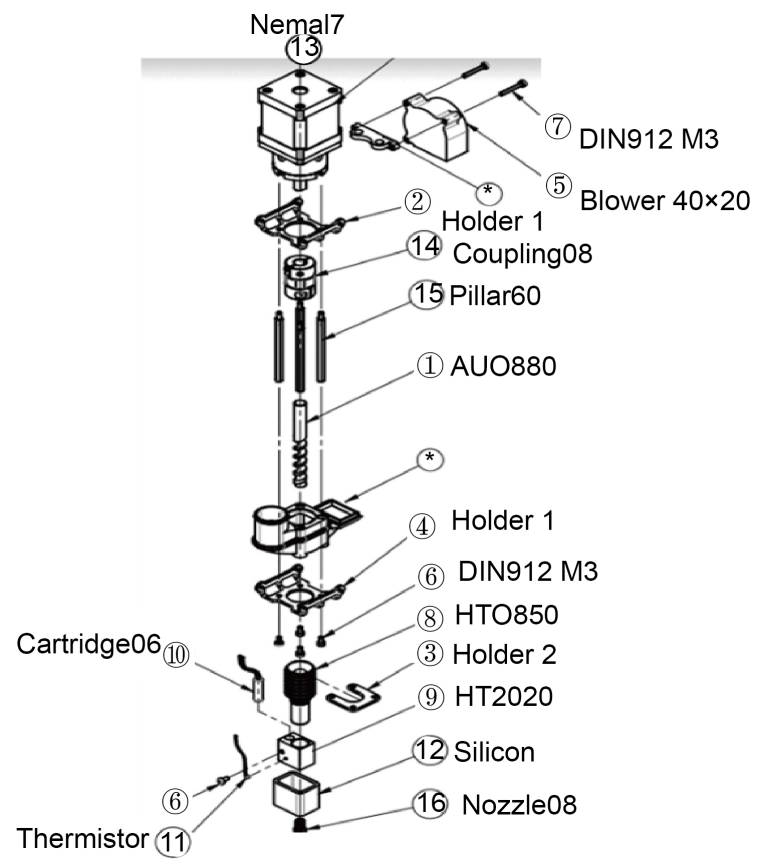

Figure 10. Pellet extruder.

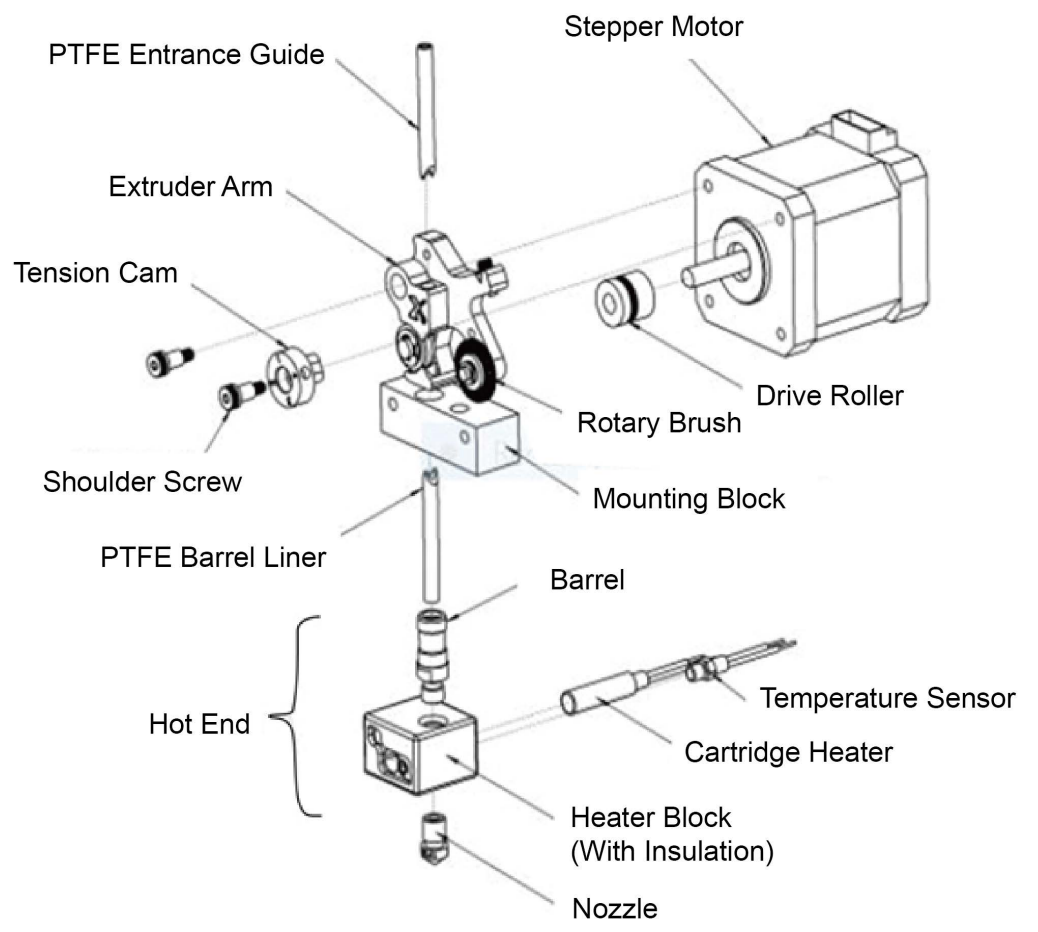

Figure 11. Filament extruder.

\subsection{Comparison of Pellet Extruder and Filament Extruder}

On comparing, details mentioned in Table 1. The advantages and disadvantages of pellet extruder (Figure 10) and filament extruder (Figure 11), both having their importance depending upon the requirement of the prints. If the requirements of final print are quality and printing of complex designs, filament 
Table 1. Important specifications of both pallet extruder and filament extruder.

\begin{tabular}{|c|c|c|}
\hline Specifications & Pellet Extruders & Filament Extruders \\
\hline Cost of extruders & Moderate & Moderate \\
\hline Cost of Raw Materials & Cheap & Costly \\
\hline $\begin{array}{l}\text { Flow rate (or) Material Output } \\
\text { from the nozzle }\end{array}$ & High & Low \\
\hline Quality of the Surface finish & Low & High \\
\hline Variety of materials can use & More & Less \\
\hline Temperatures of the extruders & High & Comparably Low \\
\hline Printing of complex designs & Inflexible & Flexible \\
\hline Reinforcement with fibres & Very good & Bad \\
\hline Cost of the final product & Low & Comparably High \\
\hline
\end{tabular}

extruder is the best option. But if cost of the final print is matters then pellet extruder is best because cost of pellets are low, and the printing time is less due to high flowrate results in time and money saving. But also, through pellet extruders can be able to print materials filled with carbon fiber, glass fiber, Kevlar etc. which are difficult in filament extruders. And more considerable advantage in pellet extruders are their recyclability though enables to use not only default prints but also waste shredded plastic, which in turn helps Eco-system. Taking some precautionary measures like taking small size pellets and pre-heating of pellets can be able to prevent defects and helps to maintain printing quality [10].

\subsection{Comparing Imporant Properties of Parts Which Are Printed with FFF and FGF}

FFF (Fused Filament Fabrication) means parts are printed with plastic filament as feedstock and FPF or FGF (Fused Pellets Fabrication or Fused Granular Fabrication) means parts are printed with pellets or granules of plastic as feedstock. From a research article published in 2020, which performed experiments to compare FFF and FGF. It uses PLA material to compare above three major areas in five different forms as feedstock that are as follows: (a) Commercial PLA filament by Form Futura for FFF (b) Recycled PLA filament made in Fablab condition for FFF (c) Commercial PLA pellets type 4043D for FGF (d) PLA pellets from recycled filament for FGF (e) Shredded PLA waste for FGF. The Article Compares some major areas which plays crucial role in additive manufacturing that are:

I. Printability

II. Printing Quality

III. Material properties and

IV. Economic performances

Printability: According to the Article, prints with the commercial filament (with diameter $1.7 \mathrm{~mm}$ ) had fewer printing defects. With the recycled Fablab 
PLA filament (diameter $1.5 \mathrm{~mm}$ ) had more printing errors. When comes to pellets, virgin PLA pellets shows significantly good performance compared to recycled pellets and recycled shredded PLA.

Printing Quality: Quality of the prints from the pellets is low due to the size of the pellets, they obtain too much heat and need more time to solidify to be able to print other layers which results sagging and print failure. There in FFF, extrusion flow rate is uniform by filament pushes the molten plastic from nozzle by inside pressure [6].

Mechanical Properties: From the Article, results from the experimental testing by comparing FFF and FGF processes. Mean average tensile strength of commercial PLA filament using FFF technique 51.76 Mpa and 57.81 Mpa with $0.5 \mathrm{~mm}$ and $0.8 \mathrm{~mm}$ nozzle sizes, respectively. For recycled PLA filament, tensile strength is $50.84 \mathrm{Mpa}$ and $58.3 \mathrm{Mpa}$ with nozzle sizes $0.5 \mathrm{~mm}$ and $0.8 \mathrm{~mm}$, respectively. When considering about pellets, using FGF technique with nozzle diameter $0.5 \mathrm{~mm}$, the tensile strength is $59.1 \mathrm{Mpa}$ for virgin PLA pellets, 60.3 Mpa for recycled PLA pellets and 52.7 Mpa for shredded recycled PLA plastic. Pelletized prints are given comparable tensile strength compared to filament technique, but mean strain value is lower with a value of $5.1 \%$ details are depicted in Table 2.

Economic Performance: Cost of commercial PLA filament is $24.96 € / \mathrm{kg}$ and PLA pellets are $8.5 € / \mathrm{kg}$. Comparing prices pellets are less in cost which helps to reduce cost of production.

FGF-printed components shows significant mechanical performance, printing time and good economical factor compared to FFF technique. Results from the experiment shows a better approach for recycling own waste to print the components [14].

\section{Some Examples of Extruders Based on Pellets/Granules}

\subsection{Universal Pellet Extruder by Mahor}

Mahor is designing different types of universal pellet extruders which are compact and lightweight extruder seems to work quite well. Here discussing one of its model Universal pellet extruder Model V4. It has compatible nozzle diameters between $0.2 \mathrm{~mm}$ and $2 \mathrm{~mm}$. HT-NTC $100 \mathrm{k}$ type of thermistor is used in this model and a heater of $12 \mathrm{v}-24 \mathrm{v}$ is used to heat the heater block which is made

Table 2. Pla material cost details.

\begin{tabular}{cccccc}
\hline & Commercial & Recycled-PLA & Virgin-PLA & Recycled-PLA \\
PLA Filament & Filament & Pellets & Shredded-PLA & \\
\hline Raw-Material cost & $25.96 € / \mathrm{kg}$ & $0.0404 €$ & $8.5 €$ & $0.0404 €$ & $0.00448 €$ \\
Printing Time & $50 \mathrm{Hrs}$ & $50 \mathrm{Hrs}$ & $33 \mathrm{Hrs}$ & $43 \mathrm{Hrs}$ & $50 \mathrm{Hrs}$ \\
Printing Energy & $0.7345 € / \mathrm{kg}$ & $0.7345 € / \mathrm{kg}$ & $0.6059 € / \mathrm{kg}$ & $0.7895 € / \mathrm{kg}$ & $0.918 € / \mathrm{kg}$ \\
Total printing cost & $26.7 € / \mathrm{kg}$ & $0.7749 € / \mathrm{kg}$ & $9.106 € / \mathrm{kg}$ & $0.8298 € / \mathrm{kg}$ & $0.9226 € / \mathrm{kg}$ \\
\hline
\end{tabular}



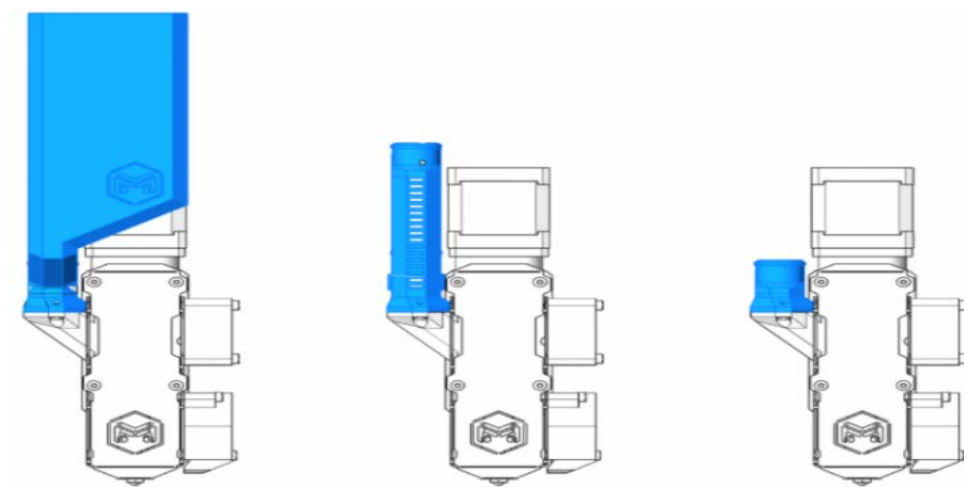

Figure 12. Mahor feeding types.

of aluminum. The variable height position allows flexibility in the configuration of the heating block to adjust the heating zone and heat transfer to the plastic, Figure 12 shows a pellet extruder.

It uses $12 \mathrm{v}-24 \mathrm{v}$ cooling fans, to reach high temperatures, around $300^{\circ} \mathrm{C}$, it is advisable to regulate the fan power by half, thus reducing cooling and allowing it to reach a higher working temperature and in less time [15].

Depending on the type of plastic or pellet size, the flow may vary so it will be necessary to adjust it using the extrusion factor. Smaller the pellet size better the result, in this model pellet not greater than $4.7 \mathrm{~mm}$ is recommended. There are several possibilities for feeding pellets through hopper:

A. Direct by gravity.

B. Automatic pellet feeder.

C. Flexible tube by gravity.

Aluminium cover for protection from accidental contact with hot surfaces, as well as fan support. They are symmetrical pieces to configure the material input hopper and fans as appropriate for each printer [16].

\subsection{DIY Direct Granules Extruder}

DIY direct granules extruder is developed by HomoFaciens. In normal conventional extruders as shown in Figure 13, the screw reaches hot zone just above the nozzle from cold zone (Feeding zone). But in DIY direct granule extruder follows unconventional method in which screw limits at cold zone as shown in Figure 14.

Pellets in cold zone are pushed hot zone. A Teflon tube is used around the screw so that granules slide well along the walls of tube to get better result. Smaller the diameter of screw better the transfer of pellets. Smaller the volume of granules moved per revolution of the extruder screw, the better control on flow of plastic coming out of nozzle. After many experiments, $0.5 \mathrm{~mm}$ diameter copper wire wrapped on a $3 \mathrm{~mm}$ screw in a Teflon tube with $5 \mathrm{~mm}$ inner diameter, the results are good. In this model, hot end is made of Aluminium with $16 \times 16$ $\mathrm{mm}$. For thermal insulation, hot end is wrapped with two layers of cardboard and exhaust sealant to glue the insulation. Speed of the nozzle ranges up to 30 


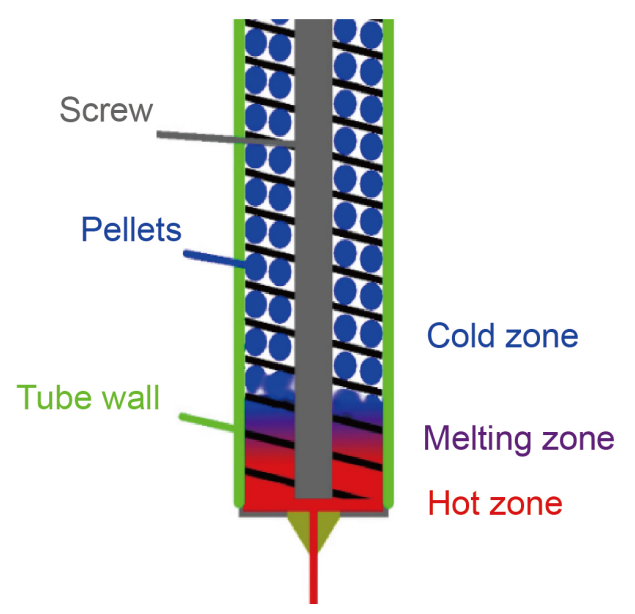

Figure 13. Conventional pellet extruder.

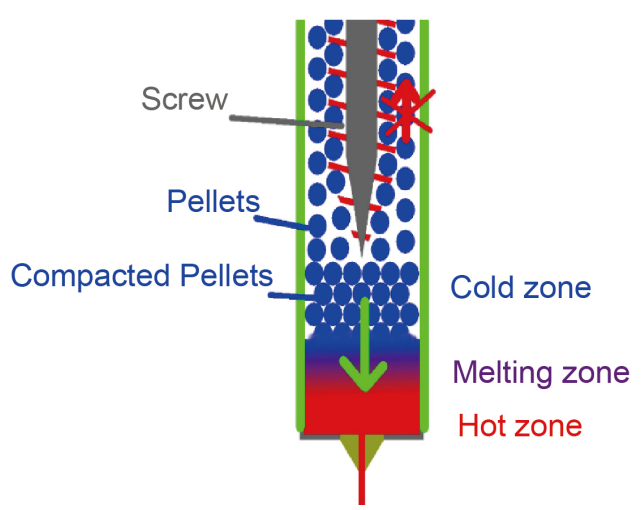

Figure 14. DIY Modified extruder.

$\mathrm{mm} / \mathrm{sec}$ and size of pellets is not greater than $2 \mathrm{~mm}$. A piece if Sheetmetal on the screw acts as a stirrer, which compacts the granules slightly before enters the hot end [17].

\subsection{Pulsar Pellet Extruder}

Pulsar pellet extruder is a fastest and can print up to $2.5 \mathrm{~kg} / \mathrm{hr}$ which is designed by Dyze Design. Not only speed it can be able to operate with large type of polymers including some high melting point polymers like PEEK and ULTEM. Polymer is maintained at constant temperature due to the presence of Tri-zone heating control design.

Pulsar pellet extruder can operate with a maximum temperature of $5000^{\circ} \mathrm{C}$ and uses three high precision class A PT100 sensors which ensure rapid melting and ensure consistent flow.

It has auto feeding system which can detect the pellet level. A strong NEMA23 geared motor is used to handle the high flow. It has $1 \mathrm{~mm}, 3 \mathrm{~mm}, 5$ $\mathrm{mm}$, nozzle sizes available so there is chance to select whether it need more resolution or more speed and all parts are made of steel because it is abrasive to prevent wearing [18]. 


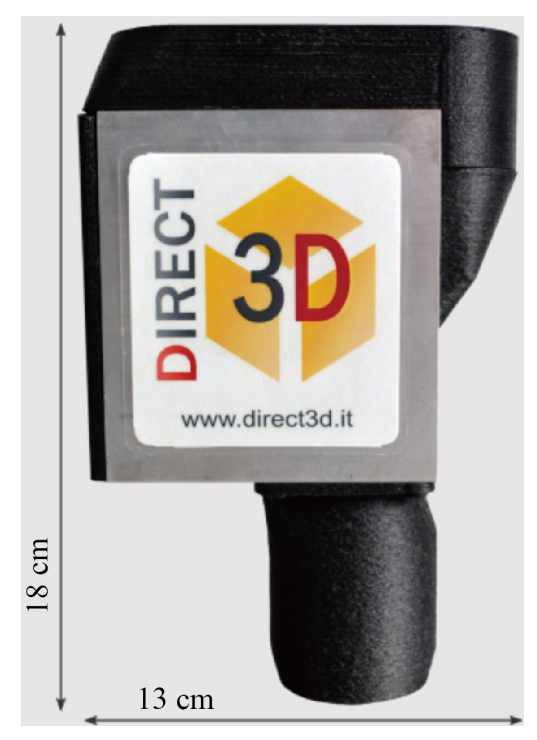

Figure 15. Direct 3D pellet extruder.

\subsection{Pellet Extruder by Direct 3D}

Pellet extruder is small and light weight about $1 \mathrm{~kg}$ and in a dimension of $18 \times 13$ $\mathrm{cm}$ as shown in Figure 15 Designed by Direct 3D. I t runs with a speed of 20 $250 \mathrm{gm} / \mathrm{hr}$ and its nozzle ranges from $0.4-2.5 \mathrm{~mm}$. Its electronic specifications as follows: with NEMA17 motor, $100 \mathrm{k}$ thermistor, heater resistor of $24 \mathrm{v}$ as well as $12 \mathrm{v}$, cooling fan of $12 \mathrm{v}-24 \mathrm{v}$ and the entire body is made of Aluminium and easy to fix all types of printers.

Many varieties of polymers are tested in this model like ABS, Polyamide, polypropylene, PE, PA-CF, PP-CF, TPU etc., It has high productivity and ideal for large parts. It operates with common slicing programs uses G-code files. Easy to install in printers like Creality, Prusa and many other printers [19].

\subsection{Pellet Extruder for Composite Thermoplastic 3D Printing}

A pellet extruder is designed especially for manufacturing composite thermoplastic using additive manufacturing by the $3 \mathrm{D}$ printer equipment manufacturer CEAD, developer of continuous fibre additive manufacturing. This extruder is whole purposely made to use on Robot Arm. It consists of $25 \mathrm{~kg}$ container with 20 meter hose, and the steady flow of pellet is achieved by blowing air from the container. Speed can reach up to $12 \mathrm{~kg} / \mathrm{hr}$ depending on material used. It uses the combination of servo motor and planetary motor to get best working of drive system.

Easy to change nozzle size, depending up on the application material nozzle size ranges from $2 \mathrm{~mm}$ to $12 \mathrm{~mm}$ and it has 4 heating zones as shown in the figure their individual temperature can controlled by using touchscreen controls. The CEAD has tested with large variety of materials that they mentioned in their website with a table which is given [20]. Table 3 shows a clear understanding of various parameters of pellet extruders. 
Table 3. Details of various pellet extruders.

\begin{tabular}{|c|c|c|c|c|c|}
\hline $\begin{array}{l}\text { Specifications } \\
\text { Extruders }\end{array}$ & UPE by Mahor & $\begin{array}{c}\text { DIY direct granule } \\
\text { extruder }\end{array}$ & Pulsar pellet extruder & Direct $3 d$ extruder & $\begin{array}{l}\text { Pellet extruder for } \\
\text { composite printing }\end{array}$ \\
\hline Nozzle range & $0.2 \mathrm{~mm}-2 \mathrm{~mm}$ & $0.4 \mathrm{~mm}-0.8 \mathrm{~mm}$ & $1 \mathrm{~mm}-5 \mathrm{~mm}$ & $0.4 \mathrm{~mm}-2.5 \mathrm{~mm}$ & $2 \mathrm{~mm}-12 \mathrm{~mm}$ \\
\hline $\begin{array}{l}\text { Maximum } \\
\text { temperature }\end{array}$ & $300^{\circ} \mathrm{C}$ & Approximately $180^{\circ} \mathrm{C}$ & $500^{\circ} \mathrm{C}$ & Approximately $250^{\circ} \mathrm{C}$ & Approximately $1200^{\circ} \mathrm{C}$ \\
\hline Weight & $0.750 \mathrm{~kg}$ & Approximately $1 \mathrm{~kg}$ & $7 \mathrm{~kg}$ & $1 \mathrm{~kg}$ & $29 \mathrm{~kg}$ \\
\hline $\begin{array}{c}\text { Speed of Material } \\
\text { Output }\end{array}$ & Up to $0.2 \mathrm{~kg} / \mathrm{hr}$ & -- & $\mathrm{Up}$ to $2.5 \mathrm{~kg} / \mathrm{hr}$ & Up to $0.250 \mathrm{~kg} / \mathrm{hr}$ & Up to $12 \mathrm{~kg} / \mathrm{hr}$ \\
\hline $\begin{array}{c}\text { Dimensions } \\
(\mathrm{L} \times \mathrm{W} \times \text { Depth })\end{array}$ & $\begin{array}{c}190 \mathrm{~mm} \times 86 \mathrm{~mm} \times 42 \\
\mathrm{~mm}\end{array}$ & -- & $\begin{array}{c}484 \mathrm{~mm} \times 166 \mathrm{~mm} \times \\
130 \mathrm{~mm}\end{array}$ & $180 \mathrm{~mm} \times 130 \mathrm{~mm}$ & $\begin{array}{c}920 \mathrm{~mm} \times 230 \mathrm{~mm} \times \\
340 \mathrm{~mm}\end{array}$ \\
\hline Cost & $496 €$ & $<150 €$ & $6679,38 €$ & $<250 €$ & $\begin{array}{l}24.900 € \text { includes } \\
\text { control system }\end{array}$ \\
\hline Manufactured with & Aluminium & Aluminium & Aluminium Alloy & Aluminium & Aluminium \\
\hline Compatible with & $\begin{array}{c}\text { Cartesian, H-bot, Core } \\
\text { XY }\end{array}$ & Cartesian type & $\begin{array}{c}\text { Cartesian, H-bot, Core } \\
\text { XY }\end{array}$ & $\begin{array}{c}\text { Cartesian, H-bot, Core } \\
\text { XY, Robot Arm }\end{array}$ & $\begin{array}{c}\text { Specially designed for } \\
\text { Robot Arm }\end{array}$ \\
\hline Materials can be use & PLA, ABS, PET, PP ... & PLA, ABS & $\begin{array}{l}\text { PLA, ABS, TPE, PSU, } \\
\text { PEEK, ULTEM, etc. }\end{array}$ & $\begin{array}{l}\text { PLA, ABS, PA, PP, PE, } \\
\text { PA-SGF, PA-CF, ETC. }\end{array}$ & $\begin{array}{c}\text { PETG } 30 \% \text { GF, PET } \\
\text { 50\% GF, ABS 20\% CF, } \\
\text { PLA/WF, ETC. }\end{array}$ \\
\hline
\end{tabular}

\section{Conclusion}

Considering all Specifications which are mentioned in the table, each extruder is the best in its own way and according to their purpose of printings product but in all of them Mahor pellet extruder is better in all specifications within an easy budget which is an affordable by all from a hobby maker to professional worker and easily compatible with our 3D printer FABMATICC which is Mendelmax FM Pro edition. The best specification of Mahor pellet extruder is its nozzle range which ranges from very low that is $0.2 \mathrm{~mm}$ which helps to maintain the quality of the print and allows retractions. The melting temperature is in a range of 300C - 3000C which enables to print with any meltable material and chance of printing with granules of filament, recycle plastic, etc., It also offers a quality print as good as filament extruder by maintaining flow rate at the nozzle, but quality and size of the pellets must be constant. Weight of the Mahor extruder is considerably very good with $0.75 \mathrm{~kg}$ which reduces the load on the carriage and helps to print fast. Moreover, it has three feeding systems like feeding of pellets like feeding by gravity, automatic pellet feeder, flexible tube by gravity. Mahor extruder nozzle is made of stainless steel which enables to print with abrasive materials [19]. From the above information even having some disadvantages in pellet extruders like bad printing quality, it is capable of printing with recycle plastic and helps in reducing the cost of production by taking pellets as raw materials. By taking some preventive measures like constant flow rate from nozzle with minimum layer width, printing quality is also achievable as good as printing in filament extruder [19]. Pellet extruder like Mahor is offering a minimum nozzle size of $0.2 \mathrm{~mm}$ with in compatible size which enables to fix maximum 3D printer, with a light weight $(0.750 \mathrm{~kg})$ body and cost $(496 €)$ is also affordable 
[19]. This has given end users the opportunity to explore their creativity: The development of screw extrusion as an alternative 3D printing process will hopefully open doors to new ideas for 3D printing. Screw extrusion will allow users to have access to a wider variety of materials with a high resolution for their $3 \mathrm{D}$ printed parts [20].

\section{Acknowledgements}

The author would like to thank Prof. Jens Schuster of University of Applied Sciences Kaiserslautern, Germany for interesting discussion on the devices and thank Mr. Aarif, without his contribution this research would have not finished.

\section{Authors' Contributions}

Mr. Yousuf \& Mr. Aarif contributed to the data collection, synthesis and writing of the initial drafts of the manuscript. Jens Schuster helped with research progress and draft revisions.

\section{Conflicts of Interest}

The authors declare no conflicts of interest.

\section{References}

[1] Varotsis, A.B. (2021) Introduction to FDM 3D Printing. 3dhubs. https://www.3dhubs.com/knowledge-base/introduction-fdm-3d-printing

[2] top3dshop. FFF vs FDM: Difference and Best Printers. top3dshop. https://top3dshop.com/blog/fff-vs-fdm-difference-and-best-printers

[3] Alex, M. (2017) 3d Printing News. 3d Natives. https://www.3dnatives.com/en/four-types-fdm-3d-printers140620174/\#

[4] O'connell, J. (2020) FDM 3D Printers Explained: Cartesian, Delta, CoreXY, \& More. All3DP.

https://all3dp.com/2/cartesian-3d-printer-delta-scara-belt-corexy-polar

[5] Zanden, T.V.D. (2016). StackExchange. https://3dprinting.stackexchange.com/questions/1519/what-are-the-parts-that-mak e-up-a-hotend-and-what-do-they-do/1522

[6] CINCINNATI (2020) Pellet vs Filament 3D Printing. https://www.e-ci.com/3d-scoop/2020/9/17/pellet-vs-filament-3d-printing

[7] Flynt, J. (2019) What to Do When Your 3D Printer Nozzle Keeps Clogging. 3DINSIDER. https://3dinsider.com/3d-printer-nozzle-clogging

[8] simplify3d (2021) simplify3d. https://www.simplify3d.com/support/print-quality-troubleshooting/stringing-or-oo zing

[9] Drotman, D., Diagne, M., Bitmead, R. and Krstic, M. (2016) Control-Oriented Energy-Based Modeling of a Screw Extruder Used for 3d Printing. 7.

[10] Boyle, B.M., Xiong, P.T., Mensch, T.E., Werder, T.J. and Miyake, G.M. (2019) 3D Printing Using Powder Melt Extrusion. 9.

[11] Jennings, A. (2021) 3D Printing Troubleshooting All Common Problems. All3DP. https://all3dp.com/1/common-3d-printing-problems-troubleshooting-3d-printer-is 
$\underline{\text { sues }}$

[12] Hart, K. (2021) Dual Extruder (3D Printing): All You Need to Know. All3DP. https://all3dp.com/2/dual-extruder-extrusion-3d-printer-simply-explained

[13] Alexandre, A., Sanchez, F.A.C., Boudaoud, H., Camargo, M. and Pearce, J.M. (2020) Mechanical Properties of Direct Waste Printing of Polylactic Acid with Universal Pellet Extruder: Comparison of Fused Filament Fabrication on Open-Source Desktop Three-Dimensional Printers.

[14] Mahor XYZ (2020) Pallet Extruder Wiki Page. Mahor XYZ. https://mahor.xyz/wiki/pellet-extruder-wiki/\#montaje

[15] bitfab (2021) Pellet Extruders for 3D Printing-Why Use Them and Which Ones Should I Buy. Mahor XYZ. https://bitfab.io/blog/pellet-extruders-3d-printing

[16] Nobert (2019) Direct Granules Extruder. Homofaciens. https://www.homofaciens.de/technics-machines-3d-printer-granule-extruder en.ht $\underline{\mathrm{m}}$

[17] Dyze Design (2020) Pulsar Large-Scale Pellet Extruders. Dyze Design. https://dyzedesign.com/pulsar-pellet-extruder

[18] Direct 3D (2018) Pellet Extruder. Direct 3D. https://www.direct3d.it

[19] Cead Composite Additive Manufacturing (2020) Pallet Extruder for Composite Thermoplastic 3D Printing. Cead Composite Additive Manufacturing. https://robotextruder.com

[20] 3D Print.me (2020) V4 Pellet Extruder. Mahor XYZ. https://3dprint.me/product/v4-pellet-extruder 